\title{
Cervico-ocular function in patients with spasmodic torticollis
}

\author{
R Stell, M Gresty, T Metcalfe, A M Bronstein
}

\begin{abstract}
The cervico-ocular (COR) and active and passive vestibulo-ocular reflexes (VOR) were measured in seven patients with spasmodic torticollis (ST) and six normal controls. The COR was found to be weak or absent in both groups. The VOR gain was similar in the two groups but five patients had a significant asymmetry of the response. There was no evidence of abnormal cervico-vestibular interaction during active head rotation. The study suggests that the VOR asymmetry frequently found in ST cannot be explained on the basis of an abnormal cervical input.
\end{abstract}

Spasmodic torticollis (ST) is the most common form of focal dystonia. Ocular motor function in idiopathic spasmodic torticollis is normal, ${ }^{12}$ apart from the presence of vestibular abnormalities. ${ }^{3-5}$ These usually take the form of a vestibulo-ocular reflex (VOR) asymmetry, with approximately $70 \%$ of patients having a more active slow phase component of induced nystagmus in the direction of chin displacement. Whilst the origin of the asymmetry is not entirely clear it does not seem to be the trivial consequence of head deviation as it persists after head posture is improved by botulinum toxin treatment, ${ }^{4}$ and when patients heads are held straight with head clamps during VOR testing. Since the slow (vestibular) component of nystagmus is faster in the direction of chin rotation, it has been argued that the VOR asymmetry reflects an underlying bias of the mechanisms involved in the control of head and eye position. ${ }^{34}$

In this paper we investigate the possibility that the vestibular asymmetry in torticollis is due to abnormal cervical proprioceptive input impinging upon central vestibular pathways, ${ }^{6-8}$ and thus influencing slow phase eye movements. It is surprising that there have been no systematic attempts to evaluate the influence of the neck receptors on the VOR in spite of the massive involvement of the neck musculature in this condition. Derangement of cervical input may occur either as a result of forced tonic head deviation with a secondary effect upon neck receptors, or alternatively, by way of descending pathways acting upon the gamma loop, and thereby influencing the sensitivity of the cervical stretch receptors. Accordingly, eye movements induced by rotating the trunk about an earth-fixed head were studied to establish the strength and symmetry of any cervical ocular reflex (COR). In addition, to investigate the possibility that neck proprioception has an effect on eye movements specifically during phasic vestibular stimulation, responses to active, head on neck rotation were assessed.

\section{Materials and methods}

Seven patients with ST, three men and four women, with a mean age of 43 years (range 25-71), were tested. Their mean disease duration was 3.8 years (range 1-6). All patients had torticollis with chin rotation to the left. None of the patients were on medications at the time of the study. Patients were compared to a group of six normal controls; one female and five males with a mean age of 35 years (range 25-60).

Subjects were seated in a motorised chair which was free to rotate about an earth vertical axis aligned approximately with the rotational axis of the neck. Eye movements were recorded with electrodes on the outer canthi using DC electronystagmography (ENG). Eye velocities were obtained by differentiation of the ENG signal using an analog differentiator tuned to the frequency content of the stimuli delivered. Chair velocity was recorded with a tachogenerator mounted on the axle and position was obtained by analog integration of the tachosignal. Head movement was recorded using an angular accelerometer mounted with its sensitive axis aligned to record rotation of the head in the plane of lateral movement of the eyes. The accelerometer was attached to the head with a helmet fitted tightly to the skull with a tourniquet. The acceleration signal was analog integrated to produce an on-line record of head velocity. Chair, head, and eye position, eye velocity and head acceleration, were displayed on an ink-jet recorder (Mingograph). Measurement of the above parameters was performed by hand directly from the paper record. Gain of the slow eye movement response was defined as the ratio between peak slow phase eye velocity and peak head/ trunk velocity. Gain to the right or left were assessed separately. Symmetry of the response was expressed using the formula: (rightwards gain - leftwards gain)/(rightwards gain + leftwards gain) $\times 100$. All subsequent experiments were carried out in the dark.

\section{STUDIES OF THE COR}

To study the role of the COR in isolation, the head was held immobilised with a dental bite 

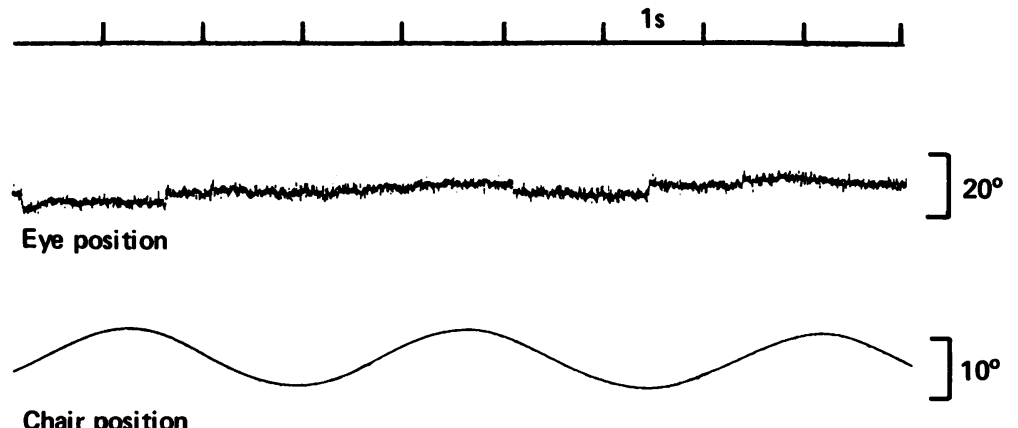

Chair position

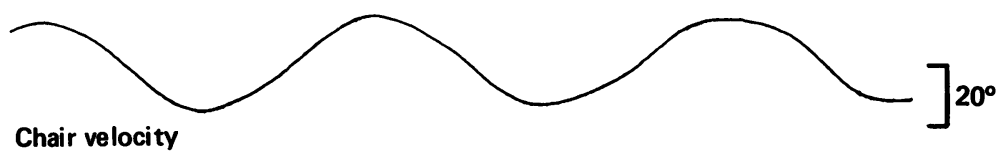

Figure 1 Eye movements during sinusoidal rotation of the trunk (chair) with the head held earth-fixed (COR) in a patient. The record demonstrates the lack of any consistent nystagmic response. For this and all subsequent illustrations rightwards motion is represented by upwards deflection of the trace and leftward motion by a downward deflection.

fixed to the wall in front of the seated subject whilst the chair, and hence the trunk, was rotated manually. Those patients who had difficulty maintaining the bite during trunk rotation were further restrained with the use of a binaural head clamp fixed to the wall. An infrared video camera was used to confirm that there was no inadvertent head movement. The chair was moved both sinusoidally and in a ramp fashion.

Sinusoidal rotation: trunk on head

An approximation to sinusoidal trunk on head oscillation was made by manual rotation of the chair in time with a metronome at a frequency of between $(0.3-0.4) \mathrm{Hz}$. Amplitudes of
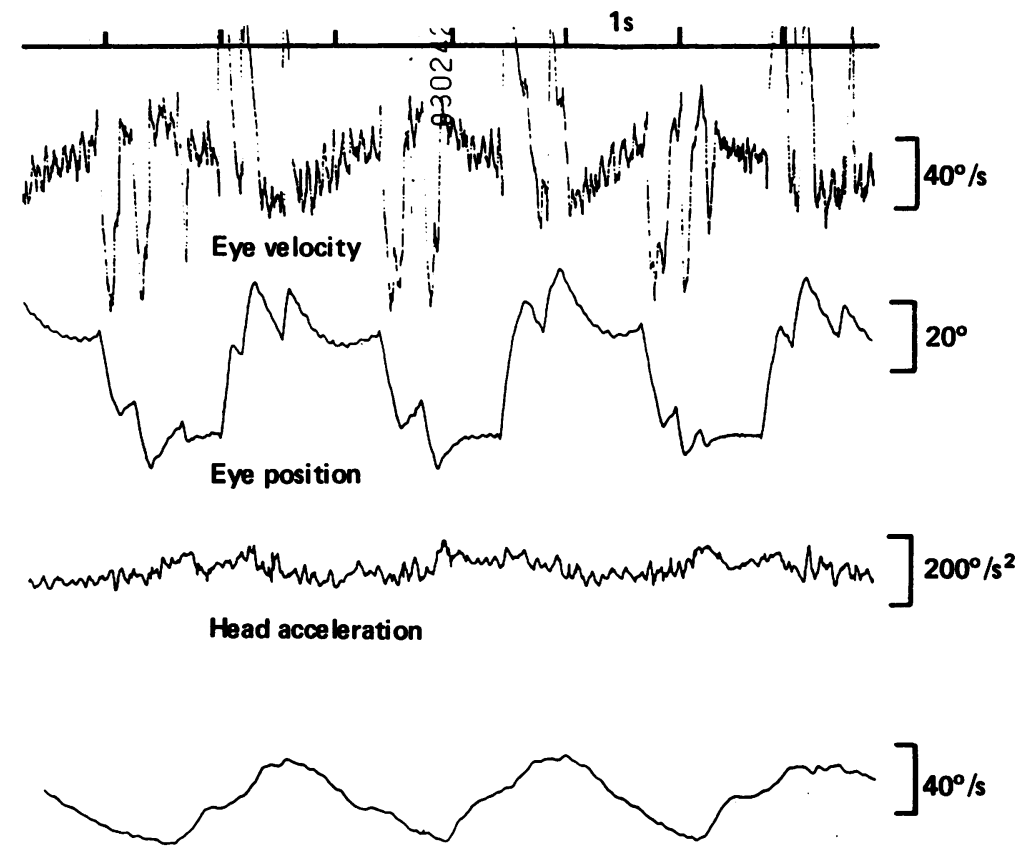

Head velocity

Figure 2 Eye movements elicited during sinusoidal rotation of the head on trunk ( $A V O R$ ) in a patient with torticollis to the left. It can be seen on the eye position trace that the slop'e of the slow phase eye movement to the left is greater than that to the right. oscillation were approximately \pm 11 to 23 degrees with peak velocities of 20 to 44 degrees/ second.

\section{Ramp stimulation: trunk on head}

In this condition the chair was moved in a ramp fashion to the right or left with peak angular displacements of between 20 and 35 degrees and peak velocities of between 10 and 22 degrees/second. The direction, amplitude and interval between steps was randomised. The subject was instructed to fixate upon a light in line with primary gaze which was briefly illuminated before each chair displacement to eliminate any residual eye deviation resulting from the preceding stimulus.

STUDIES OF THE VOR (WITH HEAD FIXED AND FREE) Active head on neck rotation (AVOR), and rotation of the head and body "en bloc" (passive VOR, PVOR) were studied. AVOR combines vestibular and neck proprioceptor stimulation whereas PVOR represents the response to a pure vestibular stimulus.

Sinusoidal stimulation: head on trunk ( $A V O R$ ) In this condition subjects were instructed to rotate their heads from right to left over amplitudes of approximately \pm 20 degrees, in time with a metronome at a frequency of $0.4 \mathrm{~Hz}$ whilst performing mental arithmetic. Some patients found it difficult to move their heads in a smooth sinusoid and were therefore guided manually to aid them in this task.

\section{Sinusoidal stimulation: head with trunk (PVOR)}

Subjects had their VOR studied in response to whole body (head with trunk) sinusoidal rotation in the dark at $0.4 \mathrm{~Hz}$ and with a peak velocity of 63 degrees/second, whilst performing mental arithmetic. The head was immobilised with binaural clamps.

\section{Results}

Cervico-ocular reflex

There was no difference in the response of patients and controls to sinusoidal or ramp, trunk on head rotation. Neither group had a definite or consistent response to the stimulus. Slow phase eye movements when they occurred were of low gain and variable in

\section{Table 1 VOR gain for patients and controls}

\begin{tabular}{lll}
\hline Patients & AVOR & PVOR \\
\hline 1 & 0.90 & 0.80 \\
2 & 0.90 & 0.87 \\
3 & 0.88 & 0.77 \\
4 & 0.95 & 0.75 \\
5 & 0.90 & 0.81 \\
6 & 0.96 & 0.81 \\
7 & 0.95 & 0.75 \\
Mean & 0.92 & 0.79 \\
Controls & & \\
1 & 1.10 & 0.74 \\
2 & 1.03 & 0.63 \\
3 & 0.88 & 0.78 \\
4 & 1.00 & 0.96 \\
5 & 1.10 & 1.00 \\
6 & 0.81 & 0.80 \\
Mean & 0.98 & 0.81 \\
\hline
\end{tabular}



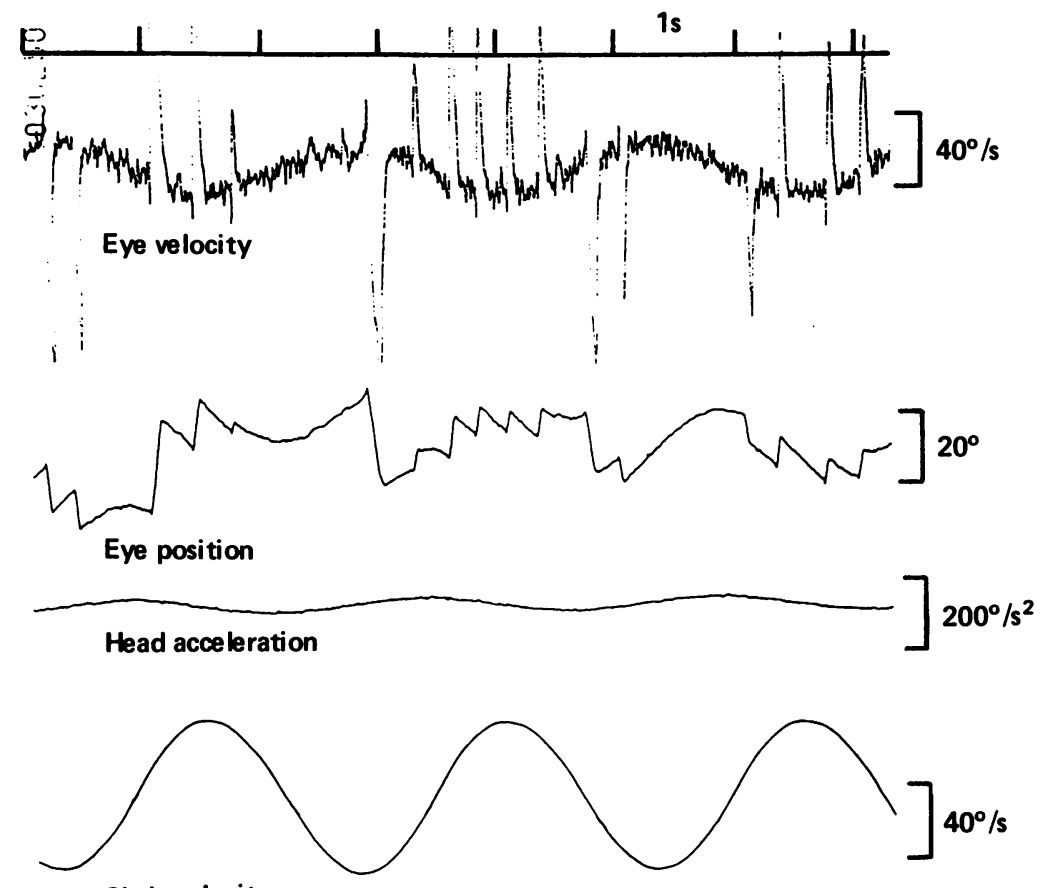

Chair velocity

Figure 3 Eye movements elicited during sinusoidal rotation of the head and trunk en bloc (PVOR), in same patient as fig 2.

direction bearing no consistent relationship to the direction of trunk rotation (fig 1). The eye movement responses for both controls and patients were the same as those of a previous study of the COR in 13 normal subjects. ${ }^{9}$

\section{Vestibulo-ocular reflex}

Sinusoidal rotation of the head on trunk (AVOR), and of the head and trunk en bloc (PVOR) in the dark, produced a consistent vestibular nystagmic response in the direction of head rotation (figs 2,3 ). Mean VOR gains (rightward and leftward combined) are presented in table 1 . AVOR gain was $17 \%$ greater than the PVOR gain in the patient group, and $20 \%$ greater in the control group. The mean gains of AVOR and PVOR were not statistically different between the two groups (AVOR: $\mathrm{t}=1.41, \mathrm{p}>0.10$; PVOR: $\mathrm{t}=0.56$, $\mathrm{p}>0 \cdot 2)$.

Five patients with torticollis had an asymmetry of both active and passive VOR, with slow phase velocities higher in the direction of chin displacement (table 2). There was no

Table 2 Slow phase asymmetry of the VOR (\%)

\begin{tabular}{|c|c|c|}
\hline Patients & $A V O R^{\star}$ & $P V O R^{\star}$ \\
\hline $\begin{array}{l}1 \\
2 \\
3 \\
4 \\
5 \\
6 \\
7 \\
\text { Mean }\end{array}$ & $\begin{array}{l}17 \\
-5 \\
-2 \\
14 \\
18 \\
21 \\
13 \\
12.9\end{array}$ & $\begin{array}{l}19 \\
-6 \\
-3 \\
12 \\
20 \\
20 \\
16 \\
13 \cdot 7\end{array}$ \\
\hline $\begin{array}{l}\text { Controls } \\
(\mathrm{n}=6) \\
\text { Mean } \\
\text { Range }\end{array}$ & $\begin{array}{l}4 \cdot 5 \\
(2-8)\end{array}$ & $\begin{array}{l}4 \cdot 0 \\
(2-7)\end{array}$ \\
\hline
\end{tabular}

significant difference in the asymmetry with respect to magnitude or direction with the head free [mean $12.9 \%$, (SD) $7 \cdot 0$ ], or fixed, [mean $13.7 \%$, (SD) 6.9]. Patients 2 and 3 had a higher slow phase VOR gain in a direction opposite to chin displacement but this did not lie outside the normal control range (table 2).

\section{Discussion}

In lower order animals the COR may play a role in the stabilisation of the eyes in space during head movements, but in primates it is virtually non-existent. ${ }^{10}$ Recent studies, however, in labyrinthectomised monkeys and patients with absent vestibular function, have shown an enhanced COR which helps to generate compensatory eye movements during head motion. ${ }^{9-11}$

The purpose of this study was to investigate the functional significance of the COR in ST, and to explore the possibility that it influences the vestibular system in such a way as to explain the VOR asymmetry found in many patients with this condition. The results demonstrate that the COR in patients with spasmodic torticollis is absent or very weak, in agreement with preliminary observations, ${ }^{12}$ and therefore cannot be held responsible for the vestibular asymmetry. In addition, head free VOR studies exclude the possibility of an abnormal phasic influence of cervical receptors upon the VOR. Our findings therefore largely exclude a peripheral cervical cause for the vestibular asymmetry in spasmodic torticollis.

These observations are consistent with the finding that the VOR asymmetry in ST is unrelated to head posture ${ }^{4}$ and add further weight to the suggestion that it is due to primary involvement of the vestibular system.

1 Huygen PLM, Verhagen WIM, Van Hoof JJM, Horstink MWI. Vestibular hyperreactivity in patients with
idiopathic spasmodic torticollis. $J$ Neurol Neurosurg idiopathic spasmodic tor

2 Stell R, Bronstein AM, Gresty M, Buckwell D, Marsden CD. Saccadic function in spasmodic torticollis. $J$ Neurol Neurosurg Psychiatry 1990;53:496-501.

3 Bronstein AM, Rudge P. Vestibular involvement in spasmodic torticollis. $J$ Neurol Neurosurg Psychiatry 1986;49:290-5.

4 Stell R, Bronstein AM, Marsden CD. Vestibulo-ocular abnormalities in spasmodic torticollis before and after botulinum toxin injections. $J$ Neurol Neurosurg Psychiatry 1989;52:57-62.

5 Diamond SG, Markham CH, Baloh RW. Ocular counterrolling abnormalities in spasmodic torticollis. Arch Neurol 1988;45:164-9.

6 Fredrickson JM, Schwartz D, Kornhuber HH. Convergence and interaction of vestibular and deep somatic afferents upon neurons in the vestibular nuclei of the cat. Acta Otolaringol (Stockh) 1966;61:168-88.

7 Brink EE, Hirai N, Wilson VJ. Influence of neck afferents on vestibulospinal neurons. Exp Brain Res 1980;38:285-92.

8 Hikosaka O, Maeda M. Cervical effects on abducens motoneurons and their interaction with vestibulo-ocular reflex. Exp Brain Res 1973;18:512-30.

9 Bronstein AM, Hood DJ. The cervico-ocular reflex in normal subjects and patients with absent vestibular function. Brain Res 1986;373:399-408.

10 Dighans J, Bizzi E, Morasso P, Tagliasco V. Mechanisms underlying recovery of eye head co-ordination following bilateral labyrinthectomy in monkeys. Exp Brain Res 1973;18:548-62.

11 Kasai T, Zee DS. Eye-head coordination in labyrinthinedefective human beings. Brain Res 1978;144:123-41.

12 Bronstein AM, Rudge P. The vestibular system in abnormal head postures and in spasmodic torticollis. In: Fahn $\mathrm{S}$, Marsden CD, Calne DB. Advances in neurology Vol 50, Dystonia 2. New York: Raven Press, 1988;493-500. 